\title{
Value for money of social assistance in FCAS: Considerations, evidence, and research priorities
}

\author{
Emily Wylde
}

February 2022

\section{Theme summary}

Value for Money (VfM) is an essential tool for balancing difficult policy and programme decisions and the trade-offs between the ' 5 Es' of economy, efficiency, effectiveness, cost-effectiveness, and equity. While many of the conceptual approaches to VfM and methods for estimation are similar in regular development programming for social protection and humanitarian cash and food assistance, these literatures have so far evolved in fairly distinct silos. There has been relatively little work so far to bring the two strands together.
In fragile and conflict-affected settings, the gaps are especially great. A lack of cost and basic programme implementation data hinders understanding of economy and efficiency, while gaps in robust evidence on outcomes and impacts further impede an analysis of effectiveness and, crucially, the trade-offs between the ' $5 \mathrm{Es}$ '. The research agenda presented here emphasises the need to build the evidence base on both costs and benefits, and to use it more intentionally for better adaptive management of programmes and policy support.

This thematic brief is a shortened version of a BASIC Research Working Paper. To explore this research theme in more detail please refer to:

Wylde, E. (2022) Value for Money of Social Assistance in Fragile Contexts: Considerations, Evidence, and Research Priorities, BASIC Research Working Paper 9, Brighton: Institute of Development Studies, DOI: 10.19088/BASIC.2022.009

A full list of the references cited in this brief can be found at the BASIC Research Zotero library:

\section{Implemented by}

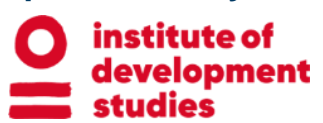

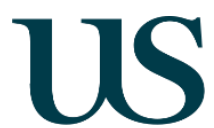

UNIVERSITY OF SUSSEX

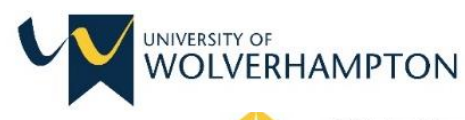

\section{CIDT}

Better Assistance in Crises (BASIC) Research (funded by UKAid) aims to inform policy and programming on how to help poor and vulnerable people cope better with crises and meet their basic needs through more effective social assistance. All costs related to BASIC Research are covered by the UK Foreign, Commonwealth and Development Office. 


\section{Understanding Value for Money in fragile contexts}

Value for Money (VfM) is fundamentally about trade-offs, often between efficiency and effectiveness, and short-term versus long-term. The starting point for understanding VfM in any setting is always the ' 5 Es': economy, efficiency, effectiveness, cost-effectiveness and equity. However, the key is to focus not only on these 'Es' in isolation, but on the balance between them, ideally considering the full stream of costs and benefits over the medium- to long-term and, crucially, how these are distributed.

The framework for VfM is already well developed for social protection; what is distinct about VfM for social assistance in fragile and conflict-affected settings (FCAS)? Perhaps the best way to frame this is in terms of the way that the calculus of costs and benefits changes in FCAS compared to stable settings. This is as a result of:

- the ways in which conflict can increase the costs of delivery, alter considerations of equity based on recognising who experiences conflict in which ways, and what that means for needs and the benefits of social assistance responses;

- the ways in which state will and capacity affect costs of delivery (if the state is unwilling or unable to deliver support) and the benefits of long-term system building;

- the role of local actors and how they might be able to improve both efficiency and equity;

- the implications of forced displacement and questions about the trade-offs between costs of immediate delivery and differences in benefit streams in displaced settings, as well as the costs and benefits of long-term systems integration;

- the implications of often short-term financing for humanitarian interventions and the (often perverse) incentives this can create to undermine good decision-making on VfM.

\section{State of the evidence and debate}

While the literature on VfM in stable contexts is fairly well developed, research on VfM in humanitarian cash programming is especially thin. What little there is focuses more on issues related to economy and efficiency, with limited discussions of issues related to effectiveness. The key themes in the existing literature have been:

- Cash versus in-kind transfers and payment modalities. Although there are often concerns that FCAS might not be 'cash ready', in most instances, cash and vouchers are both more efficient than transfers in-kind. In an overwhelming majority of cases, studies have found that delivering assistance through cash transfers or vouchers is significantly cheaper than providing aid-in-kind worth the same value; it is also more effective, giving clients the ability to spend flexibly according to their preferences.

- Multi-year funding implications. Multi-funding was found to increase economy, efficiency, and effectiveness through lower procurement costs due to better planning, better diagnostic analysis to improve delivery, greater community engagement to build trust, greater staff retention, and greater integration of gender-sensitive design elements.

With respect to the nexus, there has been some limited work to estimate the effectiveness of 'shrinking the need' through early response and resilience building. Significant gains to early response have been estimated in micro-simulations and a recent evaluation of a programme response; these found that very small delays in the timing of assistance, by even one day, had measurably reduced impacts on recipients of a one-off transfer in advance of floods. These provide powerful illustrations of the VfM of avoiding descents into poverty traps that are very difficult to escape, and the significant cost savings of early interventions. 


\section{Gaps in the evidence}

On the social protection side, some important gaps remain that are also relevant for FCAS. These include which design features and implementation models work best for which groups; how adaptive or shock-responsive programming can protect consumption and reduce negative coping strategies; what 'graduation' really means in different contexts and what pre-conditions are necessary to achieve it; and finally, what kinds of capacity development initiatives are effective under different governance conditions.

The gaps are even wider with respect to VfM in FCAS specifically, especially related to questions around effectiveness and overall VfM. Part of this gap is related to the dearth of robust evidence on outcomes and impacts of humanitarian provision. At the same time, even data that should be available on budgets and programme implementation are hard to obtain, so even analysis 'lower down the chain' on economy and efficiency is sparse.

Better understanding of heterogeneity is also essential for more nuanced and accurate VfM assessments of equity and the implications for potential trade-offs with efficiency and effectiveness: who participates and who benefits, why, and how? In particular, there are gaps in understanding gendered and socially constructed contexts in which social protection programmes operate, and the ways in which programme design and implementation can achieve better outcomes for women, people with disabilities, socially marginalised groups, and the extreme poor.

Beyond these gaps in the content, there are also important gaps in the application of VfM, even in stable contexts. VfM assessments should be an ideal way to foster initial exploration of policy questions: they should (1) be vehicles to articulate different feasible options and start to set out the trade-offs across the $5 \mathrm{Es}$, (2) document an initial set of assumptions even where concrete evidence is lacking, and (3) model the potential implications. However, in practice, VfM assessments tend to be used in more limited ways related to the demands of project cycles, with varying degrees of rigour in economic appraisals of business cases and often mechanistic reporting on efficiency metrics during annual reviews, instead of VfM being more fully integrated into adaptive management and learning approaches. 


\section{Directions for research}

The gaps in the evidence point to these directions for research:

- Making sense of cost data across the nexus. What are the key cost drivers in different contexts, and how do these compare across different programmes, implementing arrangements, and types of crises? How do these compare with the cost drivers in more stable settings (in levels and shares), and are there any implications for potential gains in efficiency and effectiveness? How are VfM metrics being used (if at all) by programme managers?

- Understanding better the potential benefit streams in different contexts and for different groups. This includes, critically, an initial baseline understanding of the impacts of conflict and complex crises on poverty of different groups (who is affected in what ways?), and what this implies regarding whom to target with interventions, and how; what kind of support packages will have the greatest impacts; and what the options are for delivering these through different mechanisms, whether humanitarian or government systems.

- Bringing these together in comprehensive cost/benefit analyses of concrete options, over the short, medium, and long term, including implications for system building. To cover:

- targeting approaches, including harmonising targeting approaches of humanitarian provision with national systems, and which approaches are most appropriate in which contexts for which groups;

- cash-plus and graduation programme elements;

- investments in government systems, and how this compares with humanitarian provision in different FCAS (refugees, conflict, etc.);

○ inclusion of grievance and redress mechanisms, localisation approaches;

- integration of Gender Equality and Social Inclusion (GESI) perspectives in a more concrete way;

- adaptive social protection programming, with early response.

- Implications of finance. What are the concrete issues related to 'money in/money out' and how do these manifest in terms of VfM considerations, including public finance management issues and implications for distribution/equity?

\section{Acknowledgements and Disclaimer}

This document was developed by the Better Assistance in Crises (BASIC) Research programme. BASIC is implemented by the Institute of Development Studies (IDS), the University of Sussex and the Centre for International Development and Training, funded by UKAid from the UK government. The views expressed in this document are entirely those of the authors and do not necessarily represent views or policies of the UK governments official policies.

(C) IDS copyright 2022. Copyright in the typographical arrangement and design rests with IDS.

This publication (excluding the logos) may be reproduced free of charge in any format or medium, provided that it is reproduced accurately and not used in a misleading context. The material must be acknowledged as IDS copyright with the title and source of the publication specified.

Published by IDS.

DOI: $10.19088 / \mathrm{BASIC} .2022 .022$ 